\title{
Community Targets of JWST's Early Release Science Program: Evaluation of WASP-63b
}

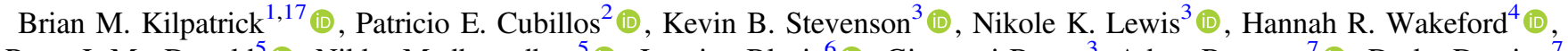

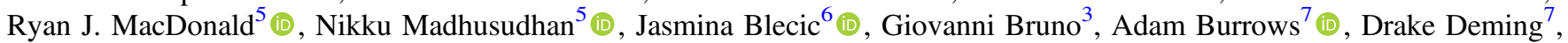
Kevin Heng ${ }^{8}$ (1), Michael R. Line ${ }^{9}$ (10), Caroline V. Morley ${ }^{10,18}$ (1) , Vivien Parmentier $^{11,18}$ (1), Gregory S. Tucker ${ }^{1}$ (D), Jeff A. Valenti ${ }^{3}$, Ingo P. Waldmann ${ }^{12}$ (1) , Jacob L. Bean ${ }^{13}$ (1) , Charles Beichman $^{14}$, Jonathan Fraine ${ }^{3}$, J. E. Krick ${ }^{15}$, Joshua D. Lothringer ${ }^{11}$ (D), and Avi M. Mandell ${ }^{16}$ (1)

${ }^{1}$ Department of Physics, Box 1843, Brown University, Providence, RI 02904, USA

${ }^{2}$ Space Research Institute, Austrian Academy of Sciences, Schmiedlstrasse 6, A-8042 Graz, Austria ${ }^{3}$ Space Telescope Science Institute, Baltimore, MD 21218, USA

${ }^{4}$ Planetary Systems Lab, NASA Goddard Space Flight Center, Greenbelt, MD 20771, USA

${ }^{5}$ Institute of Astronomy, University of Cambridge, Madingley Road, Cambridge, CB3 OHA, UK

${ }^{6}$ Department of Physics, New York University Abu Dhabi, P.O. Box 129188 Abu Dhabi, UAE

${ }^{7}$ Department of Astronomy, University of Maryland, College Park, MD 20742, USA; ddeming@astro.umd.edu

${ }^{8}$ University of Bern, Center for Space and Habitability, Sidlerstrasse 5, CH-3012, Bern, Switzerland

${ }^{9}$ School of Earth \& Space Exploration, Arizona State University, Phoenix, AZ 85282, USA

${ }^{10}$ Department of Astronomy, Harvard University, Cambridge, MA 02138, USA

${ }^{11}$ Lunar \& Planetary Laboratory, University of Arizona, Tucson, AZ 85721, USA

${ }^{12}$ Department of Physics \& Astronomy, University College London, Gower Street, WC1E6BT, UK

${ }^{13}$ Department of Astronomy and Astrophysics, University of Chicago, 5640 S Ellis Avenue, Chicago, IL 60637, USA

${ }^{14}$ NASA Exoplanet Science Institute, California Institute of Technology, Jet Propulsion Laboratory, Pasadena, CA, USA

${ }^{15}$ Spitzer Science Center, Infrared Processing and Analysis Center, California Institute of Technology, Mail Code 220-6, Pasadena, CA 91125, USA

${ }^{16}$ Solar System Exploration Division, NASA Goddard Space Flight Center, Greenbelt, MD 20771, USA

Received 2017 April 24; revised 2018 June 15; accepted 2018 June 18; published 2018 August 17

\begin{abstract}
We present observations of WASP-63b by the Hubble Space Telescope (HST) as part of "A Preparatory Program to Identify the Single Best Transiting Exoplanet for James Webb Space Telescope (JWST) Early Release Science (ERS)." WASP-63b is one of the community targets under consideration for the JWST ERS program. We present a spectrum derived from a single observation by HST Wide Field Camera 3 in the near-infrared. We engaged groups across the transiting exoplanet community to participate in the analysis of the data and present results from each. Extraction of the transmission spectrum by several independent analyses find an $\mathrm{H}_{2} \mathrm{O}$ absorption feature with varying degrees of significance ranging from $1 \sigma$ to $3 \sigma$. The feature, in all cases, is muted in comparison to a clear atmosphere at solar composition. The reasons for the muting of this feature are ambiguous due to a degeneracy between clouds and composition. The data does not yield robust detections of any molecular species other than $\mathrm{H}_{2} \mathrm{O}$. The group was motivated to perform an additional set of retrieval exercises to investigate an apparent bump in the spectrum at $\sim 1.55 \mu \mathrm{m}$. We explore possible disequilibrium chemistry and find this feature is consistent with super-solar HCN abundance but it is questionable if the required mixing ratio of $\mathrm{HCN}$ is chemically and physically plausible. The ultimate goal of this study is to vet WASP-63b as a potential community target to best demonstrate the capabilities and systematics of JWST instruments for transiting exoplanet science. In the case of WASP-63b, the presence of a detectable water feature indicates that WASP-63b remains a plausible target for JWST observations.
\end{abstract}

Key words: atmospheric effects - planets and satellites: atmospheres - planets and satellites: individual (WASP$63 \mathrm{~b})$ - techniques: spectroscopic

\section{Introduction}

The James Webb Space Telescope (JWST) will revolutionize transiting exoplanet atmospheric science due to a combination of its capability for continuous, long duration observations and its larger collecting area, spectral coverage, and resolution compared to existing space-based facilities. We previously outlined a plan in Stevenson et al. (2016) to fully demonstrate the capabilities of the JWST instruments during the Early Release Science (ERS) program allowing the community to plan more efficient and successful transiting exoplanet characterization programs in later cycles.

\footnotetext{
${ }^{17}$ NASA Earth and Space Science Fellow.

18 NASA Sagan Fellow.
}

Stevenson et al. (2016) identified a set of "community targets" that meet a certain set of criteria for ecliptic latitude, period, host star brightness, well constrained orbital parameters, and predicted strength of spectroscopic features. WASP-63b was identified as one of the strongest transmission spectroscopy candidates for JWST Early Release Science. It is an inflated planet $\left(1.43 R_{\mathrm{J}}\right)$ with a low mass of only $0.38 M_{\mathrm{J}}$ (Hellier et al. 2012) resulting in a large atmospheric scale height. It orbits a bright (11.2 $\left.V_{\mathrm{mag}}\right)$ star. Additionally, WASP$63 \mathrm{~b}$ occupies an important, underexplored region of transmission spectroscopy due to its mass. Most exoplanets studied in detail with transmission spectroscopy are either hot Jupiters of mass $\left(\sim 1-3 M_{\mathrm{J}}\right) \quad$ (e.g., Deming et al. 2013; Kreidberg et al. 2014a, 2015; Line et al. 2016; Sing et al. 2016) or Super-Earth-to-Neptune mass planets $\left(\sim 0.01-0.1 M_{\mathrm{J}}\right)$ (e.g., 
Fraine et al. 2014; Knutson et al. 2014; Kreidberg et al. 2014b). In order to understand formation and evolution processes, it's important to understand the composition of atmospheres over a full and continuous range of masses (e.g., Mordasini et al. 2016).

WASP-63b will be accessible to JWST approximately six months after the planned 2019 April start of Cycle 1 and ERS observations, making it an ideal candidate should there be any delays in the JWST timetable. Here, we observe WASP-63b to evaluate its suitability as a prime candidate to test the capabilities of JWST. We can use the strength of the water absorption feature at $1.4 \mu \mathrm{m}$ as a way to screen potential targets for the presence of obscuring aerosols and determine the amplitude of predicted spectral features (e.g., Deming et al. 2013; Kreidberg et al. 2014a; Sing et al. 2016; Stevenson 2016). Ideally, a clear atmosphere with large amplitude spectroscopic features will be best suited for benchmarking the instruments and identifying their systematics.

\section{Observations and Analysis}

We observed the WASP-63 system using the Hubble Space Telescope (HST) Wide Field Camera 3 (WFC3) on 2016 September 19. The observations were taken as part of program GO-14642, (PI Stevenson). The observations were made using the G141 grism in forward/reverse spatial scan mode. Spatial scanning (McCullough \& MacKenty 2012) involves slewing the telescope in the cross-dispersion direction during the exposure. In forward/reverse mode, the telescope is exposing in both directions of the slew thus eliminating time to reset the target at the initial position on the detector between exposures. Each exposure, utilizing SPARS10, consists of 16 non-destructive reads (NDRs) with a total exposure time of approximately $103 \mathrm{~s}$, which yielded peak per pixel counts near 32000 electrons. The spectrum was read out using the $256 \times 256$ subarray with a scan rate of $\sim 0.08 \operatorname{arcsec~s}^{-1}\left(0.62\right.$ pixels s$\left.^{-1}\right)$. This corresponds to a total scan length of $\sim 8.76$ arcsec, which spreads the spectrum in the cross-dispersion direction over $\sim 70$ pixels. We observed WASP-63 for a total of eight HST orbits, yielding a total of 86 time series integrations, to cover the entirety of the relatively long duration of transit ( $\sim 5 \mathrm{hr})$.

We use the IMA files from the CalWF3 pipeline in our analysis. These files have been calibrated for dark current and zero read bias. We applied flat field corrections to each NDR. Each NDR was background subtracted by considering a background window consisting of $(\sim 50)$ rows of pixels below the spectrum spanning the dispersion direction. A mean value for each column of the background window was taken to produce a one-dimensional (1D) background correction. The 1D solution was then smoothed in the dispersion direction to correct for outliers. The column by column background value was then subtracted from each pixel of the image. We then extract the spectrum by taking the difference between successive NDRs. We apply a top hat filter to each NDR to limit any contribution from cosmic rays and/or overlapping spectra (Evans et al. 2016; Wakeford et al. 2017). The differences between each NDR are then summed to produce a final working image.

\subsection{Band Integrated Light Curve}

We perform the extraction of the band integrated light curve (white light curve) using a range of different aperture sizes in the cross-dispersion direction. The aperture sizes range from \pm 10 pixels - \pm 10 Each orbit includes a direct image of the star from which we calculate the stellar centroid using applying a two-dimensional (2D) Gaussian fit to a $5 \times 5$ section of pixels centered on maximum pixel. The trace and wavelength solutions are calculated from the centroid of the undispersed image using the coefficients provided in Kuntschner et al. (2009). We extract a box from each working image with the number of columns corresponding to wavelength limits $1.125-1.65 \mu \mathrm{m}$ and rows determined by the chosen aperture size. The band integrated light curve is the summation of all pixels within the box at each time step. The results of the initial extraction of the raw white light curve are shown in Figure 1 (top).

Fitting the white light curve requires accounting for $H S T$ systematics. We choose to follow the standard practice of discarding the first orbit as it presents different systematics from the remainder of the data (e.g., Deming et al. 2013; Stevenson et al. 2014). The raw light curve exhibits a ramp-like increase in flux, commonly referred to as the "hook," with each HST orbit consistent with previous observations (e.g., Berta et al. 2012; Deming et al. 2013; Fraine et al. 2014; Kreidberg et al. 2014a). The hook effect, shown in Figure 1 (middle), is generally steeper in the first frame of each HST orbit, so we discard those data points. We then model the hook as an exponential plus linear function of the form $1-A \exp \left\{S\left(\theta-\theta_{0}\right)\right\}+c_{1} \theta$ where $\theta$ is the $H S T$ orbital phase, $\theta_{0}$ is a reference angle for setting zero $H S T$ phase and $\mathrm{A}, \mathrm{S}$, and $\mathrm{c}_{1}$ are scaling factors. The hook model is combined multiplicatively with a second-order polynomial in time over the entirety of the observation.

We model the transit using the methods of Mandel \& Agol (2002) implemented by the Python routine BATMAN Kreidberg (2015). The transit model assumes a circular orbit with a fixed period. Orbital parameters used for the transit model were taken from Hellier et al. (2012). We calculate nonlinear limb-darkening coefficients using the PHOENIX Code to fit theoretical spectra as described in detail in de Wit et al. (2016). We assume a stellar $T_{\text {eff }}$ of $5550 \pm 100 \mathrm{~K}$ and $\log (\mathrm{g})$ of $4.01+0.02 /-0.04$ producing coefficients $c_{1} \ldots c_{4}$ of $(0.36627439,0.63188403,-0.69135111$, $0.23393244)$ for a limb-darkening law of the form $I(\mu)=$ $I_{0}\left[1-c_{1}\left(1-\mu^{1 / 2}\right)-c_{2}(1-\mu)\right]-c_{3}\left(1-\mu^{3 / 2}\right)-c_{4}\left(1-\mu^{2}\right)$. During the fitting process, we allow for the time of transit center $\left(T_{\text {cen }}\right)$, planetary radius as a fraction of stellar radius $\left(R_{p} / R_{\star}\right), a / R_{\star}$, inclination (i), and a normalizing factor (the ratio of the amplitudes of the scan directions) to be free parameters and fit both scan directions simultaneously. All fits and uncertainty estimates are derived from the Python routine "emcee" (Foreman-Mackey et al. 2013) utilizing 50 walkers with $10^{4}$ steps. Each walker is initialized at a point in parameter space determined by randomly shifting the value of each parameter by some fraction of twice the corresponding one sigma uncertainty. This forms an over-distributed sphere of starting points surrounding the initial best-fit value. Given the number of observational data during both ingress and egress, we place uninformative priors on the orbital properties. The first $20 \%$ of steps are removed for burn in. We test for convergence using Gelman Rubin statistics with a threshold for acceptance of 1.1 and find convergence to levels of 1.01-1.03 in all cases (Gelman \& Rubin 1992). We choose the best aperture by minimizing the scatter of the residuals of the white 

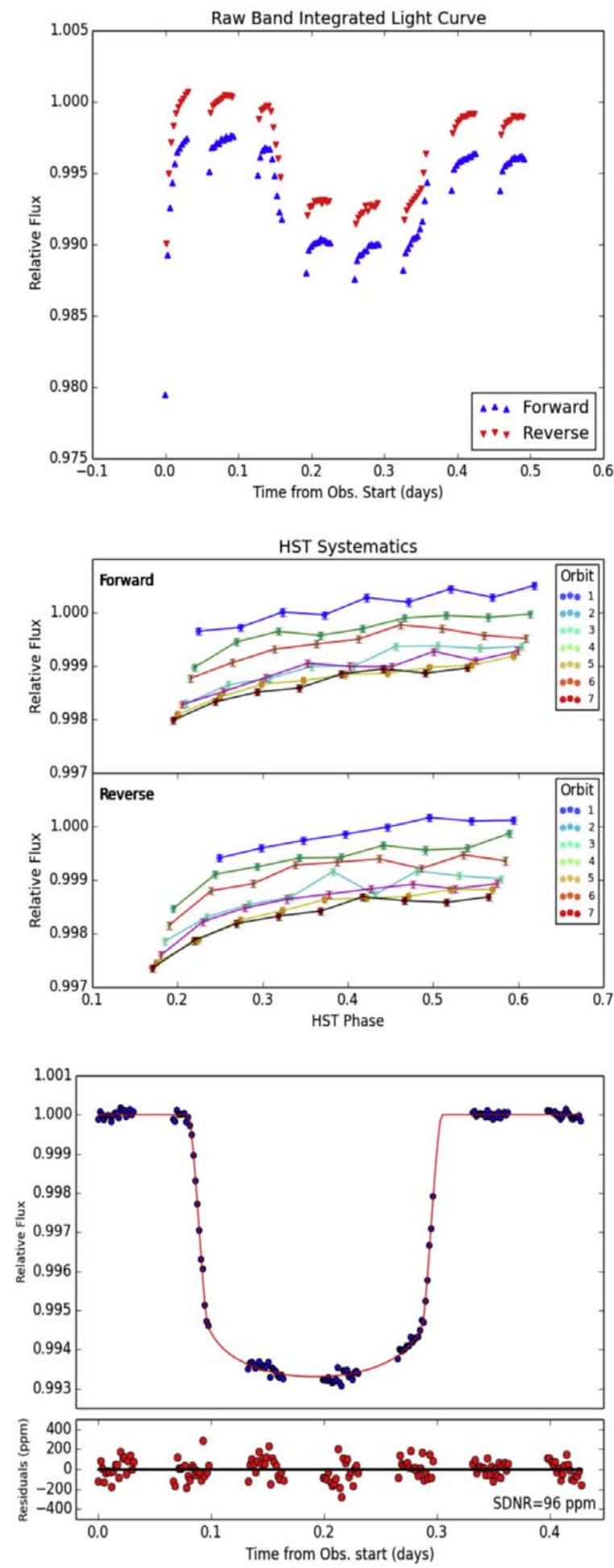

Figure 1. Top panel: the normalized raw band integrated light curve. Forward/ reverse scans are shown in red/blue. Middle panel: the Band Integrated Light Curve phase folded by HST Orbital Phase after removing the best-fit transit model to illustrate the systematic "hook" in WFC3 observations. The forward and reverse scan directions are shown in separate panels. Each color corresponds to an $H S T$ orbit. Note that here the first orbit has been discarded since it exhibits different systematics leaving only seven orbits. The exponential increase over each orbit and a visit long decrease in response are evident by visual inspection. Bottom panel: the best-fit white light curve shown with systematics removed. We achieve a standard deviation of the normalized residuals of $96 \mathrm{ppm}$.
Table 1

Best-fit Values and Uncertainties for the Planetary Physical Properties and Ephemeris from the Band Integrated Light Curve Fit

\begin{tabular}{lccc}
\hline \hline$R_{p} / R_{\star}$ & $T_{\text {cen }}(\mathrm{MJD})$ & $a / R_{\star}$ & $i\left({ }^{\circ}\right)$ \\
\hline $0.077762 \pm_{0.000183}^{0.000204}$ & $57650.435 \pm_{5.93 \times 10^{-5}}^{6.97 \times 10^{-5}}$ & $6.633 \pm_{0.015}^{0.031}$ & $88.52 \pm_{0.12}^{0.26}$
\end{tabular}

light curve fit. We find a best aperture of \pm 44 pixels in the spatial direction centered on the spectral image centroid. We achieve a standard deviation of the normalized residuals (SDNR) of 96 parts per million (ppm). There is no indication that the selection of aperture size has a statistically significant effect on the transit-depth fit. The standard deviation of the transit depth for the five apertures above and below the best aperture is $93 \mathrm{ppm}$ with an average $R p / R s$ of 0.077761 . These results all fit well within our best-fit value and uncertainty. The best-fit white light curve is shown in Figure 1 (bottom) and the values and uncertainties for the planetary physical properties and ephemeris from the best-fit white light curve are presented in Table 1.

\subsection{Spectral Light Curves}

The spectral light curves are extracted using the same aperture we found to minimize the SDNR of the white light curve. The range of wavelengths included in our aperture are divided into 15 bins of width $0.035 \mu \mathrm{m}$. The spectrum from each frame is compared to the spectrum of the first frame using cross correlation in Fourier space to check for any shift in the wavelength-pixel solution. The shift in wavelength solution throughout the observation was on the order of a few tenths of a pixel. Each column was summed and weighted by the fraction of that pixel in the bin.

The systematics were removed from the spectral light curves using the divide white method (Deming et al. 2013; Kreidberg et al. 2014a; Stevenson et al. 2014). Removing the best-fit transit from the white light curve leaves only the systematics. We divide each spectral light curve by the systematics. This assumes that the systematics are wavelength independent. We do note a linear, observation-long, wavelength dependence in the corrected spectral light curves. We use a first-order polynomial to account for the wavelength dependent systematics combined with the transit model when fitting the spectral light curves. We fix the ephemerides to the white light curve solutions and use fixed, wavelength dependent, nonlinear limb-darkening coefficients derived in the same way as described in Section 2.1. The transit depth and normalization factors are left as the only free parameters. We fit both scan directions simultaneously. Spectral light curves and their fits are shown in Figure 2.

\subsection{Transmission Spectrum}

A transmission spectrum was derived from the transit-depth fits of the spectral light curves. The change in the apparent planetary radius as a function of wavelength can be indicative of absorption features of molecular species in the planetary atmosphere. As a test for robustness, the spectrum of WASP-63b was extracted using multiple independent analysis pipelines in addition to the method described in detail in the previous subsections (Table 2). Figure 3 shows a comparison of the results from this 

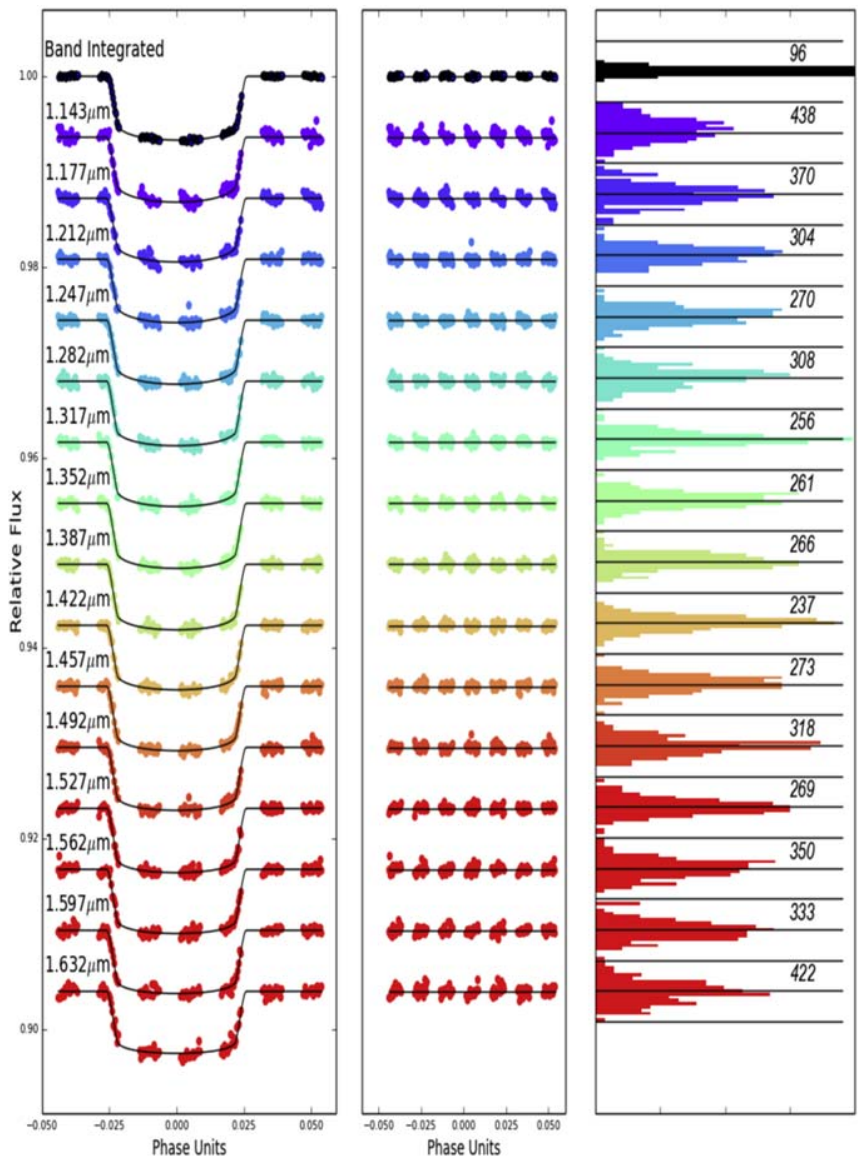

Figure 2. Left panel: spectrophotometric transit light curves (colored dots) with systematics removed compared with the best-fit transit (solid line). Light curves are shown as orbital phase (0-1 with 0 as the center of transit) vs. relative flux (vertically shifted for clarity). Each light curve is labeled with the central wavelength of the spectral bin. Center panel: residuals to the fit. Right panel: normalized histograms of the residuals. The solid black horizontal lines are spaced at $1000 \mathrm{ppm}$ intervals for scale and the SDNR of each fit is listed in units of ppm.

methodology (BMK) with that of analysis performed using methods described in Stevenson et al. (2014) (KBS) and Wakeford et al. (2016, 2017) (HRW). The spectra are in good agreement with nearly all points from each of the methods in $1 \sigma$ agreement with each of the other methods. As a test to determine how small differences in the extracted spectrum would affect retrieval results, we analyzed the KBS and HRW spectra with the Pyrat Bay model (Section 3.2.1). The KBS spectrum has a shallower bump at $1.5 \mu \mathrm{m}$, and thus, the retrieval only detected the water signature significantly (not HCN). The HRW atmospheric retrieval produced similar results to BMK, but with broader $1 \sigma$ constraints, due to the larger uncertainties of the data set (compared to BMK or KBS).

We compared these results against a flat-line model using the Bayesian Information Criterion (BIC; Liddle 2007). For the atmospheric model of each data set, we searched for the combination of free parameters that minimized BIC.

For KBS, the BIC favors the atmospheric model $\left(\mathrm{BIC}=24.59\right.$, with three free parameters, $T, R_{0.1 \mathrm{bar}}$, and H_2O), against the flat-line model $(\mathrm{BIC}=30.82$, one free parameter). The posterior odds give the flat-line model a fractional probability of only 0.04 .

For HRW, the BIC favors again the atmospheric model $\left(\mathrm{BIC}=19.19\right.$, with four free parameters, $T, R_{0.1 \mathrm{bar}}, \mathrm{H} \_2 \mathrm{O}$, and
HCN) over the flat-line model $(\mathrm{BIC}=22.92)$. However, this time there is weaker evidence, with the flat-line model having a fractional probability of 0.13 .

Therefore, in all three data sets, there is evidence of spectral features, but with different degrees of confidence. The BMK spectrum shows strong evidence, KBS spectrum shows moderate evidence, whereas HRW shows only weak evidence for water absorption. The HRW spectrum is based upon the marginalization technique described in Wakeford et al. (2016) and represents the most conservative estimate of uncertainty. As such, it serves as a sort of lower bound on the detection threshold whereas the BMK and KBS methods assume we are justified in our choice of systematic model. The arguments for this choice of model are well established and summarized in Section 2.1 and so we will choose the BMK spectral extraction to continue on with retrieval analysis.

\section{Results}

The results produced by the methodology described in in detail in Section 2 (presented in Table 2 as BMK) were distributed to the members of the transiting exoplanet community who were involved with the preparation of Stevenson et al. (2016) and/or HST program GO 14642. Each was given an opportunity to provide an independent analysis of the results. Wide community involvement resulted in a number of contributions in the form of forward model comparisons and retrievals. Here we present the methods and findings from each interpretation of the BMK spectrum. All models in this section adopt the system parameters from Hellier et al. (2012).

\subsection{Forward Models}

\subsubsection{Burrows}

We apply the transit models from Howe \& Burrows (2012), which adopt chemical-equilibrium abundances for molecular species from Burrows \& Sharp (1999) and opacities from Sharp \& Burrows (2007). The atmospheric models consider an isothermal temperature profile and gray haze opacity with cross sections of $0.001-0.005 \mathrm{~cm}^{2} \mathrm{~g}^{-1}$ from $10^{-6}$ bar to 1 bar.

By exploring a range of temperatures, haze opacities, metallicities, and nonequilibrium $\mathrm{CO} / \mathrm{CH}_{4}$ abundances, the best-fitting solutions pointed to solar-abundance atmospheres at a temperature of $1000 \mathrm{~K}$, with a haze/cloud muting the water absorption feature at $1.4 \mu \mathrm{m}$ (Figure 4 , top panel). There is no indication of significant $\mathrm{CH}_{4}$. There is a slight degeneracy between the cloud thickness and temperature, but it is clear that the atmosphere is cloudy. These models do not show a significant metallicity dependence. Finally, a high CO abundance excess $(\sim 100$ times solar) can help to fit the data at $1.5 \mu \mathrm{m}$, but it does not seem realistic.

\subsubsection{Heng}

As a complementary approach to the full retrieval calculations, we fit the data with a three-parameter analytical model (Heng \& Kitzmann 2017). In that study, it was demonstrated that this isothermal, isobaric model matched full numerical calculations at the $\sim 0.1 \%$ level over the WFC3 wavelength range. The model has three parameters: temperature, water abundance, and a constant cloud opacity. The constant cloud opacity assumes that the cloud particles are large over the wavelength range probed by WFC3 (i.e., micron-sized or larger 
Table 2

Transmission Spectrum of WASP-63b Measured with HST WFC3 G141 Grism

\begin{tabular}{|c|c|c|c|c|c|c|c|c|}
\hline \multicolumn{3}{|c|}{ BMK } & \multicolumn{3}{|c|}{ KBS } & \multicolumn{3}{|c|}{ HRW } \\
\hline Wavelength $(\mu \mathrm{m})$ & $R_{p} / R_{\star}$ & $\overline{\text { Unc (ppm) }}$ & Wavelength $(\mu \mathrm{m})$ & $R_{p} / R_{\star}$ & $\overline{\text { Unc (ppm) }}$ & Wavelength $(\mu \mathrm{m})$ & $R_{p} / R_{\star}$ & $\overline{\text { Unc (ppm) }}$ \\
\hline 1.1425 & 0.07791 & 310 & 1.1425 & 0.07819 & 290 & 1.1352 & 0.07810 & 460 \\
\hline 1.1775 & 0.07732 & 290 & 1.1775 & 0.07801 & 275 & 1.1677 & 0.07740 & 450 \\
\hline 1.2125 & 0.07717 & 300 & 1.2125 & 0.07785 & 290 & 1.2002 & 0.07762 & 430 \\
\hline 1.2475 & 0.07753 & 300 & 1.2475 & 0.07770 & 270 & 1.2327 & 0.07839 & 420 \\
\hline 1.2825 & 0.07812 & 280 & 1.2825 & 0.07848 & 260 & 1.2651 & 0.07832 & 420 \\
\hline 1.3175 & 0.07789 & 270 & 1.3175 & 0.07846 & 285 & 1.2977 & 0.07782 & 420 \\
\hline 1.3525 & 0.07847 & 290 & 1.3525 & 0.07853 & 265 & 1.3301 & 0.07832 & 420 \\
\hline 1.3875 & 0.07891 & 300 & 1.3875 & 0.07861 & 270 & 1.3626 & 0.07921 & 420 \\
\hline 1.4225 & 0.07832 & 290 & 1.4225 & 0.07826 & 290 & 1.3951 & 0.07880 & 425 \\
\hline 1.4575 & 0.07839 & 300 & 1.4575 & 0.07849 & 280 & 1.4275 & 0.07848 & 440 \\
\hline 1.4925 & 0.07773 & 330 & 1.4925 & 0.07791 & 290 & 1.4600 & 0.07862 & 445 \\
\hline 1.5275 & 0.07865 & 330 & 1.5275 & 0.07831 & 295 & 1.4925 & 0.07811 & 450 \\
\hline 1.5625 & 0.07866 & 370 & 1.5625 & 0.07833 & 320 & 1.5250 & 0.07908 & 455 \\
\hline 1.5975 & 0.07816 & 350 & 1.5975 & 0.07752 & 310 & 1.5575 & 0.07892 & 485 \\
\hline 1.6325 & 0.07751 & 360 & 1.6325 & 0.07718 & 320 & 1.5899 & 0.07821 & 490 \\
\hline & & & & & & 1.6224 & 0.07789 & 505 \\
\hline
\end{tabular}

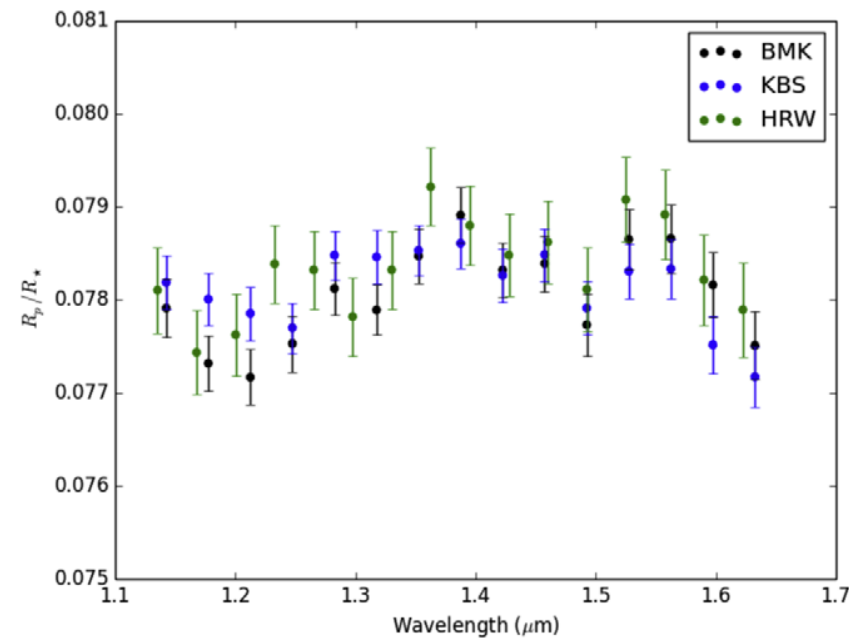

Figure 3. Results of spectral extraction from multiple independent analyses show good agreement. Here we show the best-fit value for $R_{p} / R_{\star}$ as a function of wavelength with $1 \sigma$ error bars derived from the MCMC posteriors. Colors correspond to analysis performed by Brian Kilpatrick (BMK), Kevin Stevenson (KBS), and Hannah Wakeford (HRW).

radius). Water opacities are computed using the HELIOS-K opacity calculator (Grimm \& Heng 2015) and the HITEMP spectroscopic database. This procedure confronts the data with a simple model, which has a minimal number of parameters, to serve as a plausibility check.

Following the approach of Kreidberg et al. (2015), we equate the reference transit radius to the white-light radius and set the reference pressure to $10 \mathrm{bar}$. We assume a hydrogen-dominated atmosphere and set the mean molecular weight to 2.4. The top panel of Figure 4 shows the best-fit model to the WFC3 WASP-63b data. Our general conclusion mirrors that of the retrieval calculations: water is present in the atmosphere of WASP-63b, but its presence is muted by a continuum, which in this case is attributed to a constant cloud opacity. The values of our fitting parameters span a temperature range from 500 to $1000 \mathrm{~K}$, a water mixing ratio from $\sim 10^{-8}$ to $10^{-7}$, and a cloud opacity $\sim 10^{-8}$ to $10^{-7} \mathrm{~cm}^{2} \mathrm{~g}^{-1}$.

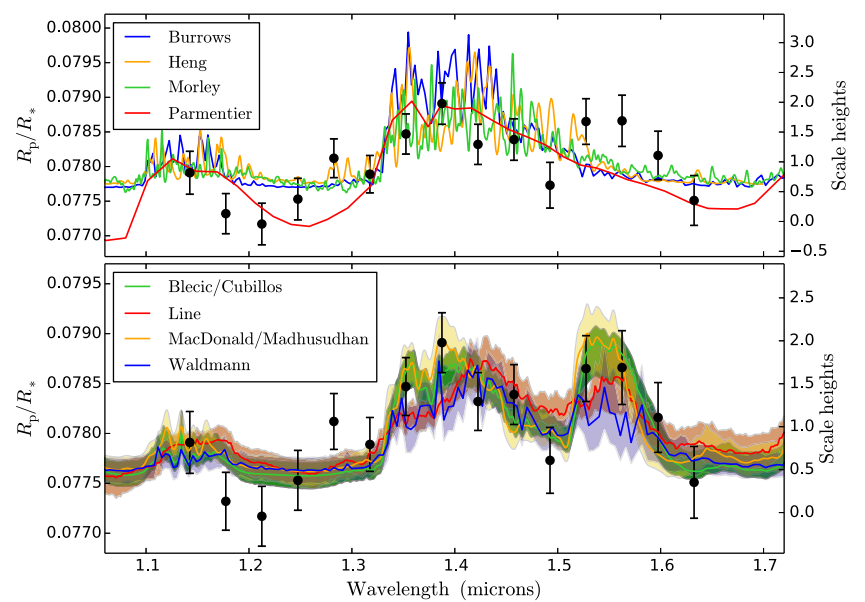

Figure 4. Top panel: WASP-63b spectrum and forward models. The black dots with error bars denote the observed best-fit radius ratio and $1 \sigma$ uncertainties. The labeled solid curves show representative forward model fits to the data described in Section 3.1. Bottom panel: WASP-63b spectrum and retrieval models including $\mathrm{HCN}$ absorption. The black dots with error bars denote the observed best-fit radius ratio and $1 \sigma$ uncertainties. The labeled solid curves denote the best-fitting models for the consistent retrieval run described in Section 4 . The vertical shaded areas around each model denote the span of the $1 \sigma$ confidence region of the posterior distribution of sampled models.

\subsubsection{Morley}

In order to determine the clouds that are predicted to form in the atmosphere of WASP-63b and their effect on the planet's transmission spectrum, we ran self-consistent models including the effects of cloud condensation. These models solve for the temperature structure of the atmosphere in radiative-convective and chemical equilibrium and are more extensively described in McKay et al. (1989), Marley et al. (1996, 1999, 2002), Burrows et al. (1997), Fortney (2005), Saumon \& Marley (2008), Fortney et al. (2008), Morley et al. (2015). Our opacity database for gases is described in Freedman et al. $(2008$, 2014), and we calculate the effect of cloud opacity using Mie theory, assuming spherical particles. We include iron and silicate clouds and vary the cloud sedimentation efficiency $f_{\text {sed }}$ from 0.1 to 1 , and find that these clouds do indeed form at high altitudes and damp the size of the signal for low sedimentation 
efficiencies (i.e., lofted clouds of small particles). Figure 4 top panel shows a representative transmission model for WASP-63b.

\subsubsection{Parmentier}

In order to understand how the three-dimensional structure of the planet might affect our interpretation of the planet's transmission spectrum, we model WASP-63b with the threedimensional global circulation model SPARC/MITgcm described in Showman et al. (2009). Our model solves for the three-dimensional temperature structure of the atmosphere assuming a cloud-free, solar-composition atmosphere. We then use the temperature map to predict the position of the clouds at the limb of the planet by comparing the partial pressure and the saturation pressure of the cloud gaseous constituents as described in Parmentier et al. (2016). The cloud-top level and size of the cloud particles are free parameters representing vertical mixing and microphysics respectively. We then compute the transmission spectrum of the whole atmosphere by combining the transmission spectrum obtained with the temperature and cloud profile at each latitude around the limb (Parmentier et al. 2016).

Our global circulation model predicts a temperature difference of $400 \mathrm{~K}$ between the east and west limb at the 10 mbar level. As a consequence some cloud species are predicted to be condensed all over the limb of the planet whereas others should condense only on the morning limb and be evaporated on the other one, leading to a partially cloudy atmosphere (Line \& Parmentier 2016). We computed models assuming the presence of enstatite clouds $\left(\mathrm{MgSiO}_{3}\right)$ or manganese sulfide clouds (MnS) corresponding to a fully cloudy and partially cloud case respectively. Our best-fit spectrum with enstatite clouds is very similar to the Morley model of Figure 4. It has a cloud-top pressure of 1 mbar and no constraints on the particle size. Our best-fit model with $\mathrm{MnS}$ clouds is the Parmentier model of Figure 4 . It has a limb that is $\approx 60 \%$ cloudy, resulting in a qualitatively different spectrum than the other, one-dimensional models shown here. For this model, the cloud-top pressure is $\approx 0.1$ mbar and the particle size is $\approx 1 \mu \mathrm{m}$. We conclude that the atmosphere of WASP-63b is unlikely to be clear with a solar-composition abundance of water. Both fully cloudy and partially cloudy atmospheres can exist, depending on the cloud composition. A higher signal-to-noise spectrum should be able to disentangle between the two scenarios.

\subsection{Retrievals}

Four groups provided atmospheric retrieval analyses for WASP-63b. The following subsections describe the retrieval procedure and the individual exploration from each group. Figures relative to the individual retrievals are included in the Appendix.

\subsubsection{Blecic \& Cubillos}

To model the atmosphere and spectrum of WASP-63b, we use the Python Radiative Transfer in a Bayesian framework (Pyrat Bay) package $^{19}$ (J. Blecic et al. 2018, in preparation; P. E. Cubillos et al. 2018, in preparation). Pyrat Bay is an opensource reproducible code, based on the Bayesian Atmospheric Radiative Transfer package (Blecic 2016; Cubillos 2016). The

\footnotetext{
${ }^{19}$ http://pcubillos.github.io/pyratbay
}

code provides a line-by-line radiative-transfer and a thermochemical-equilibrium abundances (Blecic et al. 2016) module, which can be use in forward or retrieval mode, via a Differential-evolution MCMC sampler (Cubillos et al. 2017). The atmospheric model consist of a 1D set of concentric shell layers, in hydrostatic equilibrium, spanning from $10^{-8}$ to 100 bar. For the temperature profile we consider either the three-channel Eddington approximation parameterization (TCEA; Line et al. 2013b) or an isothermal profile.

The Pyrat Bay code considers molecular opacities for $\mathrm{H}_{2} \mathrm{O}$ from HITEMP (Rothman et al. 2010), $\mathrm{NH}_{3}$ and $\mathrm{CH}_{4}$ from HITRAN (Rothman et al. 2013), and HCN from Exomol (Barber et al. 2014). We compressed these line-by-line data files using the REPACK package (Cubillos 2017) to extract only the strong lines that dominate the spectrum, speeding up the radiative-transfer calculation. Additionally, Pyrat Bay considers collision induced absorption (CIA) from $\mathrm{H}_{2}-\mathrm{H}_{2}$ (Borysow et al. 2001; Borysow 2002) and $\mathrm{H}_{2}-\mathrm{He}$ (Borysow et al. 1988, 1989; Borysow \& Frommhold 1989); and $\mathrm{H}_{2}$ Rayleigh scattering (Lecavelier Des Etangs et al. 2008). We also consider two cloud parameterizations, a simple gray-cloud opacity with constant cross section $\left(\mathrm{cm}^{-2}\right.$ molec $\left.^{-1}\right)$ below $10^{-5}$ bars, and a thermal-stability cloud model based on the approach described in Ackerman \& Marley (2001) and Benneke (2015), with additional flexibility (J. Blecic et al. 2018 , in preparation). We compute the opacity for either Fe or $\mathrm{MgSiO}_{3}$ condensates using Mie-scattering theory (Toon \& Ackerman 1981).

The retrieval parameterization includes free scale factors for the mixing ratios of $\mathrm{H}_{2} \mathrm{O}, \mathrm{NH}_{3}, \mathrm{CH}_{4}$, and $\mathrm{HCN}$ (verticalconstant values) and the mean molecular mass of the atmosphere; either the isothermal temperature or the $\kappa, \gamma$, and $\beta$ parameters of the TCEA model (see Line et al. 2013b); the gray-cloud cross section or the Mie-scattering cloud profile shape, condensate mole fraction, particle-size distribution, and gas number fraction just below the cloud deck.; and the planetary radius at 0.1 bar.

We explored several cases, obtaining qualitatively good fits in all gray-cloud, complex-cloud, and clear-atmosphere cases. As expected for transmission spectroscopy, the retrieval returned largely unconstrained parameters for the TCEA temperature model, suggesting that the data does not justify more complex models than an isothermal profile. In all cases, the MCMC favors lower temperatures $(T<1000 \mathrm{~K})$ than equilibrium temperature $(1500 \mathrm{~K})$ at the pressures probed by the observations. We constrain the water abundance, ranging from solar to $\sim 0.1$ times solar values (Figure 6 ). The observed water absorption feature is muted relative to a clear atmosphere with solar abundances. This is caused by a sub-solar water abundance, an absorbing cloud opacity, or a high mean molecular mass, which reflects in a strong correlation between the water abundance and the cloud cross section. When we compare retrievals with the gray and complex-cloud model, we find similar best-fitting spectra between the two cases. The complex-cloud retrieval does not constrain any of the cloud parameters when we set all four species abundances free. In the case when we set the water abundance as the only abundance free parameter, we find a somewhat better condensate-fraction constraint. Since the cloud opacity dominates only a limited region of the observed spectrum $(\sim 1.2-1.3 \mu \mathrm{m})$, we conclude that there is no need for a more complex-cloud model for this study. The Reproducible Research Compendium for the Pyrat 
Bay models is available at https://github.com/pcubillos/ KilpatrickEtal2018_WASP63b.

\subsubsection{Line}

We use the CHIMERA transmission model (Line et al. 2013a; Kreidberg et al. 2014a, 2015; Swain et al. 2014; Greene et al. 2016; Line et al. 2016). For transit geometry, the code solves the radiative-transfer equation for parallel rays across the terminator of the planet (Brown 2001; Tinetti et al. 2012). The code integrates atmospheric opacities from either correlated-K or sampled "line-by-line" absorption cross sections. To explore the parameter space, the transmission model is coupled with the PyMultiNest (Buchner et al. 2014) multimodal nested-sampling algorithm (Feroz \& Hobson 2008). The atmosphere is in hydrostatic equilibrium with heightdependent gravity, temperature, and molecular weight. The temperature profile comes from either the radiative-equilibrium model from Guillot (2010) or an isothermal profile, spanning from $10^{-7}$ to 30 bar. The atmosphere uses "thermochemically consistent" molecular abundances (as defined in Kreidberg et al. 2015), computed using the NASA CEA2 model for given elemental abundances (Lodders 2009).

The CHIMERA code considers molecular opacities for $\mathrm{H}_{2} \mathrm{O}$, $\mathrm{CH}_{4}, \mathrm{CO}, \mathrm{CO} 2, \mathrm{NH}_{3}, \mathrm{Na}, \mathrm{K}, \mathrm{TiO}, \mathrm{VO}, \mathrm{C}_{2} \mathrm{H}_{2}, \mathrm{HCN}$, and $\mathrm{FeH}$ (Freedman et al. 2014); CIA from $\mathrm{H}_{2}-\mathrm{H}_{2}$ and $\mathrm{H}_{2}-\mathrm{He}$ Richard et al. (2012); a Rayleigh power-law haze (Lecavelier Des Etangs et al. 2008); and either an opaque gray patchy cloud model (Line \& Parmentier 2016) or a more complex, Miecloud model (Lee et al. 2013).

The retrieval parameterization includes the metallicity $[\mathrm{M} / \mathrm{H}]$, the carbon-to-oxygen ratio $\log (\mathrm{C} / \mathrm{O})$, and the carbon- and nitrogen-species quench pressures (Kreidberg et al. 2015; Morley et al. 2017) to set the elemental abundances; either the isothermal temperature or the $\kappa_{\mathrm{IR}}, \gamma_{v}, T_{\text {irr }}$ parameters of Guillot (2010); the top-pressure boundary and a "patchy terminator" parameter for the gray patchy cloud model, or the $Q_{0}$ and $r$ (see Lee et al. 2013) and profile shape (mixing ratio, cloud base pressure, pressure fall off index; M R. Line et al. 2017, in preparation) for the Mie-cloud model; and a radius scale factor to set the reference altitude at 10 bar.

The "chemically consistent" retrieval detects the water spectral feature at $3.6 \sigma$ confidence (Figure 7). This is consistent with a hard upper limit on $\mathrm{C} / \mathrm{O}$ near 1 . The water band is muted relative to solar composition. The retrieval posterior shows two "composition" modes: low metallicity $([\mathrm{M} / \mathrm{H}] \lesssim$ $1.3(20 \times))$ degenerate with a cloud and high metallicity (peak near $\sim 300 \times$ solar). There is a turn-over degeneracy in cloud top versus $[\mathrm{M} / \mathrm{H}]$ (due to the effect on the mean molecular weight) resulting in the bi-modal marginalized metallicity distribution. Clouds can be present, but are not required to fit the spectra as given by the Bayes factor $(0.45)$ and result in a much lower value for the low-metallicity mode $(<0.1 \times$ solar $)$, while the high-metallicity mode remains. The highest of the sampled metallicities (greater than $\sim 50$ times solar) are possibly implausible given mass and radius of WASP-63b.

Further tests found negligible variations when imposing a temperature prior or no patchy-cloud factor. A comparison between correlated- $\mathrm{K}$ and line-by-line sampling opacities produced nearly identical results. Likewise, the more complex Mie-cloud model produced qualitatively similar main conclusions (with unconstrained cloud particle sizes, vertical extent, or cloud composition).

\subsubsection{MacDonald \& Madhusudhan}

We use the nested-sampling retrieval algorithm POSEIDON (MacDonald \& Madhusudhan 2017) to analyze the WFC3 observations of WASP-63b. The code pre-computes line-byline molecular cross sections following the methodology of Hedges \& Madhusudhan (2016) and \& Madhusudhan (2017). To compute detection significances we conduct nested Bayesian model comparisons. For simplicity, we model the atmosphere assuming an isothermal temperature-pressure profile, with 100 layers uniformly spaced in log-pressure from $10^{-6}$ to $100 \mathrm{bar}$, in hydrostatic equilibrium.

The POSEIDON code considers molecular opacities for $\mathrm{H}_{2} \mathrm{O}$ from HITEMP (Rothman et al. 2010) and $\mathrm{CH}_{4}, \mathrm{NH}_{3}$, and $\mathrm{HCN}$ from Exomol (Tennyson et al. 2016); CIA from $\mathrm{H}_{2}-\mathrm{H}_{2}$ and $\mathrm{H}_{2}-\mathrm{He}$ (Richard et al. 2012); Rayleigh scattering (Lecavelier Des Etangs et al. 2008); and a uniform-in-altitude gray-opacity cloud model.

The retrieval parameterization includes free scale factors for the mixing ratio of $\mathrm{H}_{2} \mathrm{O}, \mathrm{CH}_{4}, \mathrm{NH}_{3}$, and $\mathrm{HCN}$; the isothermal temperature; the gray-cloud opacity; and the reference pressure at the transit radius (Figure 8).

The model comparison test marginally prefer the grayopacity case $\left(\chi_{\text {red }}^{2}=1.21\right)$ over a cloud-free case $\left(\chi_{\text {red }}^{2}=1.46\right)$, with a Bayes factor of 2.2. Adopting the gray-opacity model, we detect $\mathrm{H}_{2} \mathrm{O}$ at $4.0 \sigma$ (Bayes factor $=601$ ), $\mathrm{HCN}$ at $3.1 \sigma$ (Bayes factor $=27.6)$, and "nitrogen chemistry" (combination of $\mathrm{HCN}$ and $\mathrm{NH}_{3}$ ) at $3.3 \sigma$ (Bayes factor $=53.7$ ). We do not detect $\mathrm{CH}_{4}$.

\subsubsection{Waldmann}

We retrieved the HST/WFC3 spectrum of WASP-63b using the Tau-REx atmospheric retrieval framework (Waldmann et al. 2015a, 2015b; Waldmann 2016). Based on the Tau code transmission forward models by Hollis et al. (2013), Tau-REx employs Nested Sampling (Feroz \& Hobson 2008) to solve the full Bayesian argument. Tau-REx can use high-resolution absorption cross section or correlated-k tables as opacity inputs. Here we used the latter but find both to yield equivalent results for the wavelength ranges and sensitivities of the data at hand. We include pressure-dependent line broadening where such information is available, taking into account the $\mathrm{J}$ quantum number dependence on pressure broadening coefficients. In this study, we assume an isothermal atmospheric temperaturepressure profile, spanning from to $10^{-9}$ to $10 \mathrm{bar}$.

The Tau-REx code considers molecular opacities for $\mathrm{H}_{2} \mathrm{O}$, $\mathrm{CH}_{4}, \mathrm{NH}_{3}, \mathrm{HCN}$, TiO, and VO from Exomol (Tennyson \& Yurchenko 2012), CO and CO2 from HITEMP (Rothman et al. 2010), and $\mathrm{O}_{2}, \mathrm{O}_{3}, \mathrm{NO}_{2}, \mathrm{NO}, \mathrm{HCOOH}, \mathrm{C}_{2} \mathrm{H}_{6}$, and $\mathrm{C}_{2} \mathrm{H}_{2}$ from HITRAN (Rothman et al. 2009, 2013); CIA from $\mathrm{H}_{2}-\mathrm{H}_{2}$ (Borysow et al. 2001; Borysow 2002) and $\mathrm{H}_{2}-\mathrm{He}$ (Abel et al. 2012); Rayleigh scattering (Lecavelier Des Etangs et al. 2008); and clouds using a hybrid model of gray-cloud opacities and a phenomenological Mie scattering (Lee et al. 2013).

We run two types of retrievals, a "free" retrieval with abundances of $\mathrm{H}_{2} \mathrm{O}, \mathrm{CH}_{4}, \mathrm{CO}, \mathrm{CO}_{2}, \mathrm{NH}_{3}$, (Figure 9) as well as a chemical-equilibrium retrieval using an implementation of the ACE model by Agúndez et al. (2012), with the $\mathrm{C} / \mathrm{O}$ ratio and 

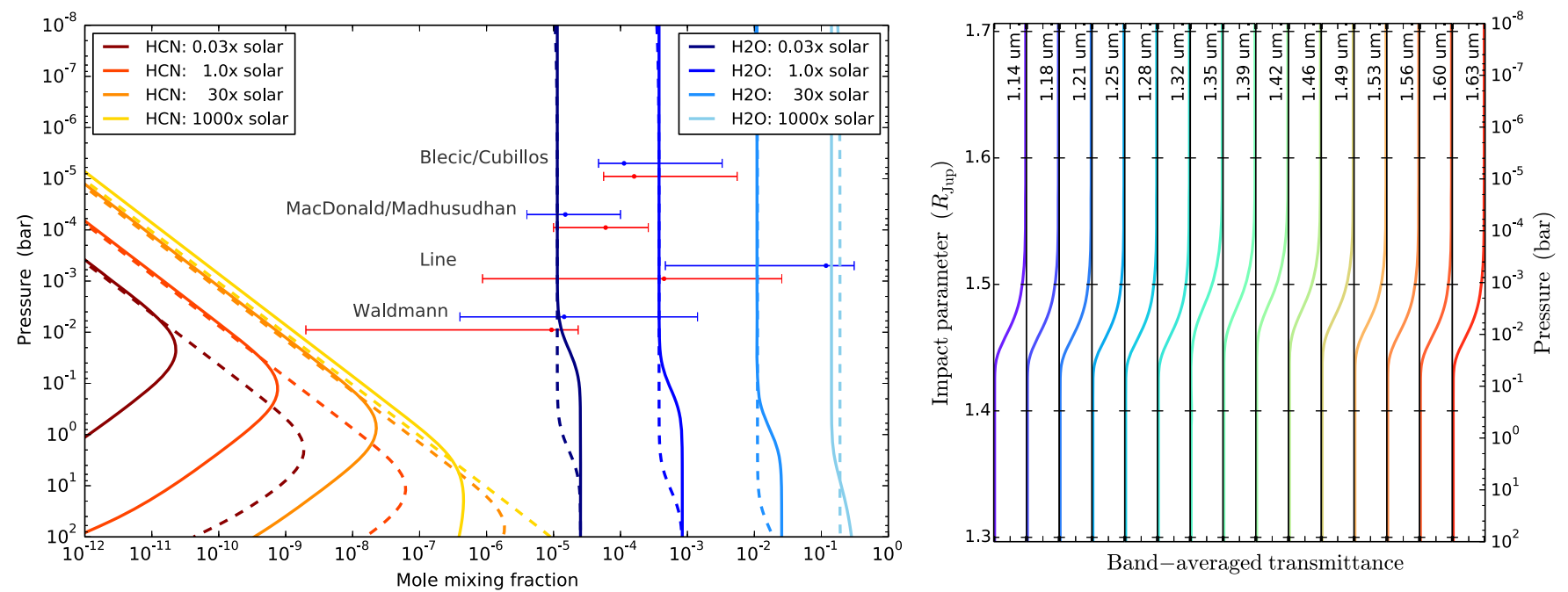

Figure 5. Left panel: water and HCN abundances for WASP-63b compared to thermochemical-equilibrium mole mixing fractions models for an isothermal atmosphere at $1000 \mathrm{~K}$ (solid lines) and at $1500 \mathrm{~K}$ (dashed lines) at a range of metallicities (see labels). The horizontal error bars show the retrieved 68\% credibleregion abundances from each group (labels) for water (blue) and HCN (red). Note that the retrieved abundances are vertically offset for clarity; see right panel for the probed pressures. All abundances correspond to the pressure levels probed by this transmission observation $\left(10^{-2}-10^{-4}\right.$ bar). All retrieved water abundances are consistent with solar or slightly sub-solar water abundances; however, the retrieved $\mathrm{HCN}$ values range several order of magnitudes higher than any $1000 \mathrm{~K}$ equilibrium value (particularly for the solar to sub-solar $\mathrm{HCN}$ curves). The retrieved mole mixing fractions of $\mathrm{HCN}$ are more plausible if the temperature at deeper layers were $\sim 1500 \mathrm{~K}$ and vertical transport dominated the abundances in the region probed by observations. The models suggest it would be possible under these conditions to produce mole mixing fractions on the order of $10^{-6}$. Right panel: sample transmittance curves as a function of impact parameter for each WFC3 pass band (see labeled wavelengths) for the Blecic/Cubillos retrieval best fit. The transition from optically thin to optically thick between $\sim 1.5$ an $\sim 1.4 R_{\mathrm{Jup}}$, respectively, denotes the photosphere of the planet, as observed by WFC3. The pressure scale on the right side denotes the deepest atmospheric pressure probed by each impact parameter. The transmittance curves from other groups show a similar trend.

atmospheric metallicity as free parameters. The rest of the retrieval parameters are the isothermal temperature; the top pressure of the gray-cloud model and the particle size, composition and mixing ratio of the Mie-cloud model; and the planet reference radius at 10 bar.

We detect water with a $3.5 \sigma$ significance (Bayes factor $=103$ ) compared with a family of pure-cloud or featureless atmosphere models. We obtain $\log \left(\mathrm{H}_{2} \mathrm{O}\right)=-4.84_{-1.53}^{+1.04}$. We do not find any evidence of an extended Rayleigh curve due to hazes but found a gray-cloud model to be sufficient. We constrain the cloud-top pressure to $\log (p)=3.08_{-0.93}^{+1.48} \mathrm{~Pa}$. The chemically consistent retrieval yielded two results. The first result yields a high-metallicity atmosphere at 370 times solar. The second result yields a low-metallicity atmosphere at 0.24 times solar. Both solutions feature comparable log-evidences and result in upper bounds of $\mathrm{C} / \mathrm{O}$ at 0.49 . A ratio of $\mathrm{C} / \mathrm{O}<0.7$ is expected as only water is retrieved in this data set and is therefore consistent with the "free" retrieval approach above. The atmospheric metallicity is poorly constrained due to the presence of clouds, which has the effect of muting the water feature and biasing the chemical-consistent model to either compensate with unrealistically high mean molecular weight atmospheres or unrealistically low trace gas abundances.

\section{Discussion}

The individual atmospheric analyses of WASP-63b agree that there is a robust water detection $(3.5-4.0 \sigma)$, but with a muted absorption feature when compared to a clear solarcomposition atmospheric model. It is unclear if the reason for the muting of the feature is the result of sub-solar water abundance, absorbing cloud opacity, or a high mean molecular mass. Thermochemically consistent retrievals show a multimodal solution due to degeneracies between cloud opacity and composition (Figures 7 and 9). Retrievals with simple temperature (isothermal) and cloud (gray-opacity) models both produced fits consistent with retrievals with more complex models and thus the data does not warrant the incorporation of more complex models nor does it allow further constraints on cloud properties.

The high transit-depth values between 1.5 and $1.6 \mu \mathrm{m}$ motivate the inclusion of $\mathrm{HCN}$ and the exploration of disequilibrium chemistry. Each retrieval team performed an additional retrieval exercise with a common set of assumptions to further explore the the inclusion of $\mathrm{HCN}$ as a means to fit the "bump" in the spectrum between 1.5 and $1.6 \mu \mathrm{m}$. We implemented an isothermal temperature model, a gray-opacity cloud model, a free pressure-radius reference point, and opacities from $\mathrm{H}_{2}$-Rayleigh, $\mathrm{H}_{2}-\mathrm{H}_{2}$ and $\mathrm{H}_{2}-\mathrm{He}$ CIA, and $\mathrm{H}_{2} \mathrm{O}, \mathrm{CH}_{4}, \mathrm{HCN}$, and $\mathrm{NH}_{3}$. We adopted molecular abundances either from thermochemically consistent calculations or from free abundances (constant vertical profiles), with the exception of the HCN abundance, which is always a free fitting variable (constant vertical profile).

We begin with this common set of assumptions and then compare retrieval results from teams with differing retrieval frameworks. Notable differences include: the statistical sampler from Blecic/Cubillos (MCMC) differ from the rest (Nested sampling), the molecular opacity handling from Waldmann and Line (correlated-K) differ from the others (cross section sampling), and the chemistry from Line (thermochemically consistent) differs from the rest (free constant vertical profiles). Figure 4 shows the retrieved spectrum from the run using the common assumptions. All four retrievals produced consistent spectral fits, seen in the intersecting 68\% confidence regions around the best-fitting models. In terms of the atmospheric characterization, these retrievals confirm the previously found water detection. 
The Bayesian hypothesis testing favors the fit with $\mathrm{HCN}$, improving the fit at $1.5-1.6 \mu \mathrm{m}$. However, the detection significance is low and inconsistent, 3.1 $\sigma$ (Blecic/Cubillos), $2.1 \sigma$ (Line), $3.1 \sigma$ (MacDonald/Madhusudhan), and $1.9 \sigma$ (Waldmann). Therefore, for the currently available data, the inclusion of $\mathrm{HCN}$ is not statistically justified within this model parameterization. Furthermore, to reproduce the observed values requires the HCN mole fraction be $\gtrsim 10^{-5}$; much higher than thermochemical-equilibrium values at the pressures probed by the WFC3 observations (Figure 5). To produce such high HCN abundances, one would need to invoke disequilibrium-chemistry processes by either quenching or photochemistry. Quenching can occur when higher temperatures at deep layers, below the levels probed by this observation, enhance the $\mathrm{HCN}$ abundance without needing the high metallicities from Figure 5. If vertical mixing dominates the mid-altitude abundances of the WASP-63b atmosphere (expected at the retrieved temperatures of $\sim 1000 \mathrm{~K})$, HCN could be effectively quenched, maintaining the high abundances from the deep layers throughout the probed region. Similar deviations from equilibrium chemistry have been modeled for other Jupiter-like exoplanets (Moses et al. 2011; Venot et al. 2012). None of the retrievals constrain any of the other molecular abundances that could provide additional evidence for quenching (e.g., $\mathrm{CO}, \mathrm{CH}_{4}, \mathrm{NH}_{3}$ ). However, photochemistry could play a role in removing these other molecules from the atmosphere while enhancing the mole fraction of $\mathrm{HCN}$ at pressures less than a millibar. Moses et al. (2011, 2013) show that ammonia and methane can be photochemically converted to $\mathrm{HCN}$ at the pressure levels probed by near-IR transmission spectroscopy thus driving the retrieved abundances much higher than equilibrium values. Future observations with extended wavelength ranges and higher sensitivity, such as JWST, can help to definitively confirm or rule out the detection of $\mathrm{HCN}$, and other atmospheric species, thus constraining the presence of disequilibrium chemistry.

\section{Conclusions}

We present the observations of one transit of the hot Jupiter WASP-63b. Observations were conducted in the near-infrared using HST WFC3 G141. This study was done as a preliminary evaluation of the suitability of WASP-63b as one of the community targets for JWST ERS science. We have detected a muted water absorption feature at $\sim 1.4 \mu \mathrm{m}$ confirming WASP$63 \mathrm{~b}$ as a potential target for ERS science. The potential presence of an absorption feature at $1.55 \mu \mathrm{m}$ is not evidence enough to make strong conclusions about the presence of other molecules in the atmosphere; however, further observations by JWST would be able to identify additional spectral features that would allow us to further constrain the atmospheric composition. The observational window for observing WASP-63b with JWST is from September 23-April 5. JWST is currently scheduled to launch in 2018 October and ERS observations would commence in 2019 April. Assuming the mission remains on schedule, WASP-63b would not be observable until several months after the ERS program window. However, if there are any delays to launch or the start of ERS observations, WASP$63 \mathrm{~b}$ would be a prime candidate for study with multiple instruments and modes.

This work is based on observations made with the NASA/ ESA Hubble Space Telescope, obtained from the Data Archive at the Space Telescope Science Institute, which is operated by the Association of Universities for Research in Astronomy, Inc., under NASA contract NAS 5-26555. These observations are associated with program GO-14642. B.M.K. acknowledges funding by HST-GO-14642.047 provided by the Space Telescope Science Institute. H.R.W. acknowledges support from the NASA Postdoctoral Program, administered by USRA through a contract with NASA. J.B. is supported by NASA trough the NASA ROSES-2016/Exoplanets Research Program, grant NNX17AC03G.

The authors acknowledge the contributions and support from members of the transiting exoplanet community who have contributed to and/or supported Program GO-14642 including: E. Agol, D. Angerhausen, T. Barman, J. Barstow, N. M. Batalha, S. Birkman, D. Charbonneau, N. Cowan, N. Crouzet, S. Curry, J. M. Desert, D. Dragomir, J. Fortney, A. Garcia Munoz, N. Gibson, J. Gizis, T. Greene, J. Harrington, T. Kataria, E. Kempton, H. Knutson, L. Kreidberg, M. Lopez-Morales, M. Rocchetto, E. Schlawin, E. Shkolnik, A. Shporer, D. Sing, K. Todorov, and J. de Wit.

\section{Appendix}

Here we present the retrieval results as described in Section 3. We present the pairs plots and fit to the observations in each case. In the case of the Line and Waldmann results, we show both the thermochemically consistent run along with the free retrieval for comparison. 


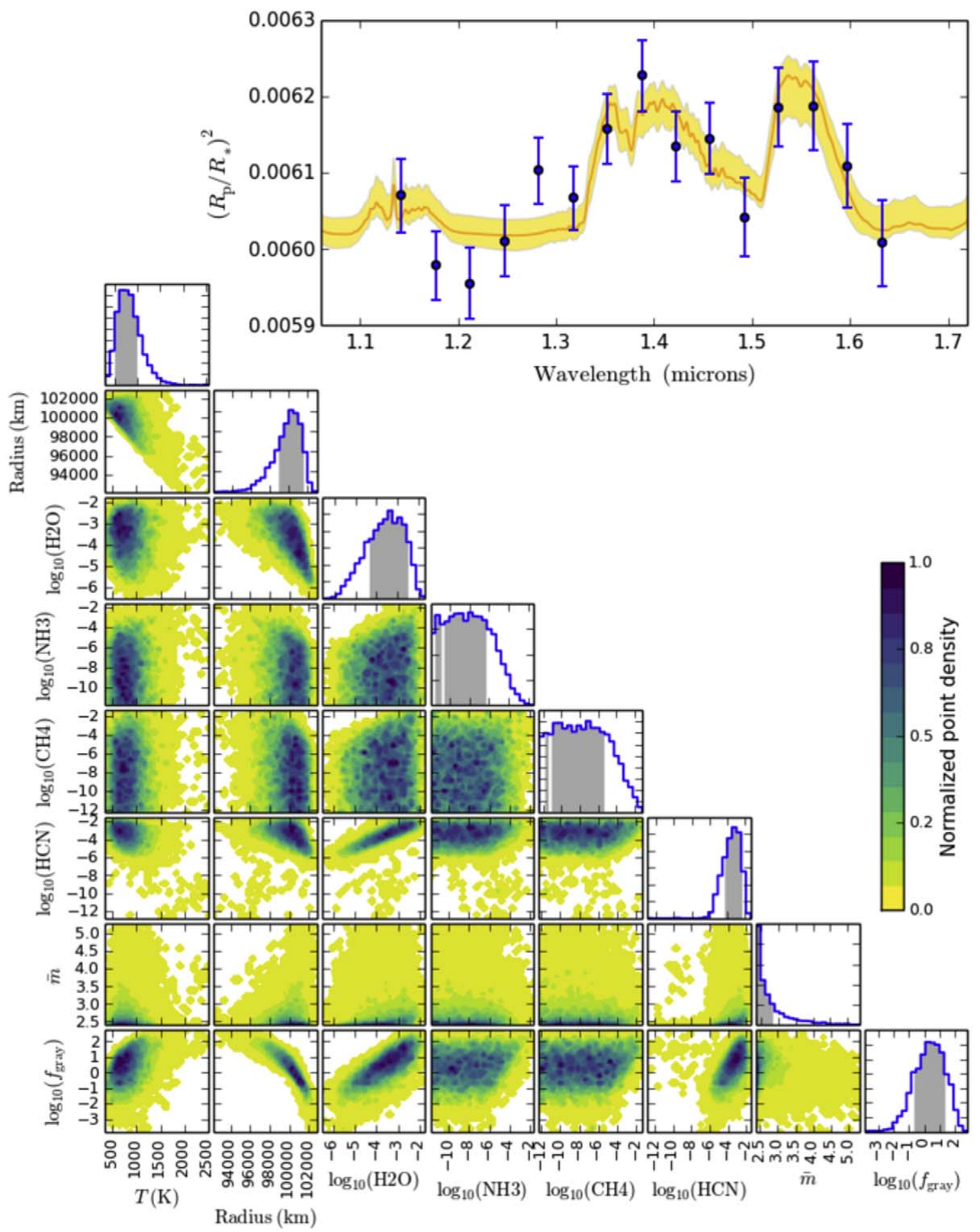

Figure 6. Posteriors and fit from retrieval parameterized by free abundances of $\mathrm{H}_{2} \mathrm{O}, \mathrm{NH}_{3}, \mathrm{CH}_{4}$, and $\mathrm{HCN}$ along with the mean molecular mass of the atmosphere. The atmosphere is assumed to be isothermal ( $T$ as a free parameter) with a gray cloud (opacity as free parameter). 


\section{LINE}

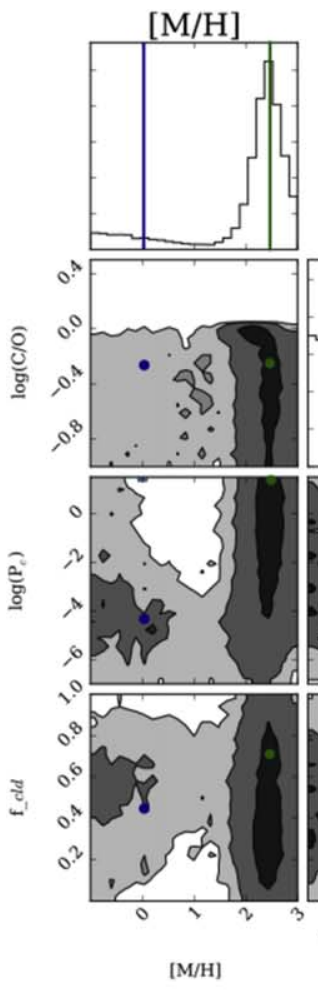

$[\mathrm{M} / \mathrm{H}]$

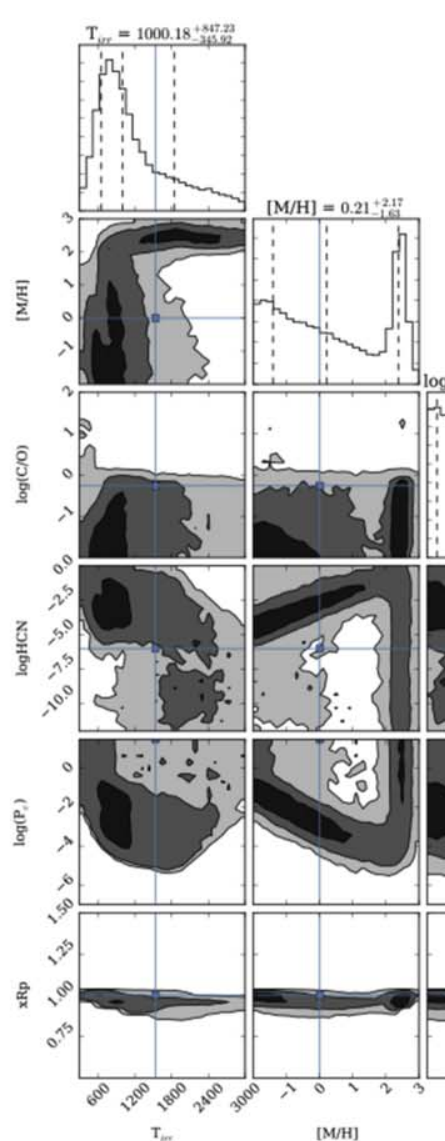

$\log (\mathrm{C} / \mathrm{O})$
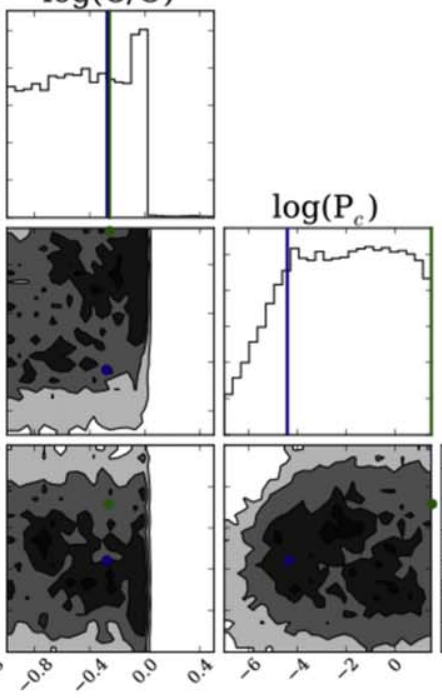

$\log \left(\mathrm{P}_{c}\right)$
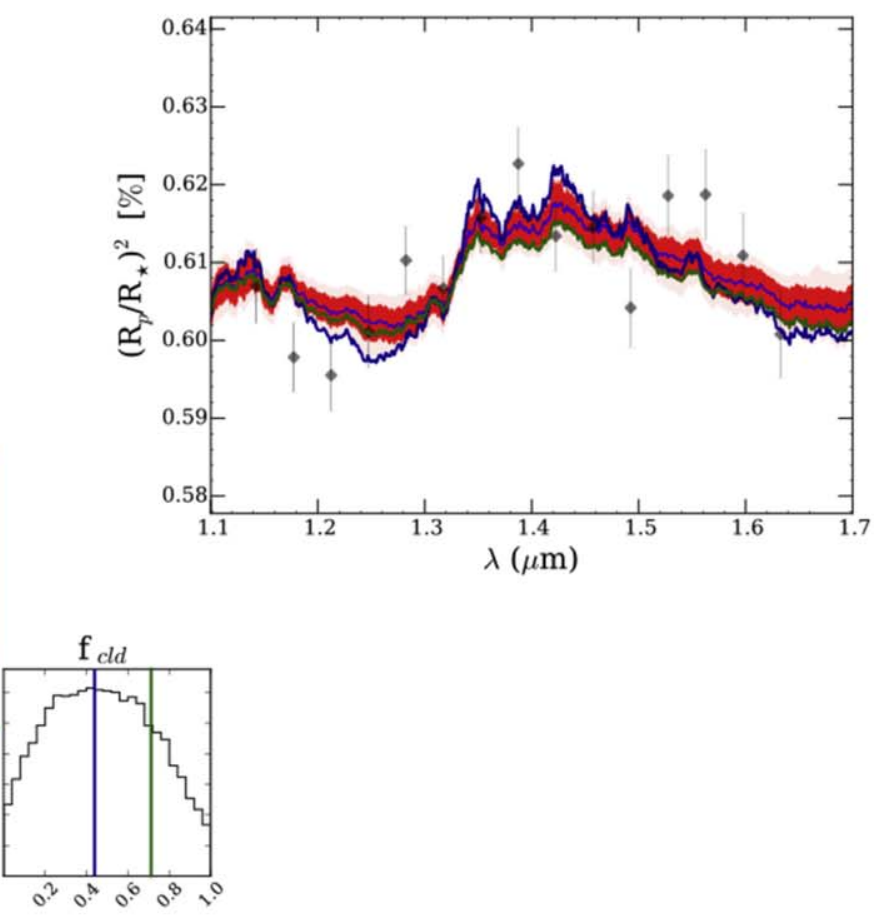

$f, \cdot$

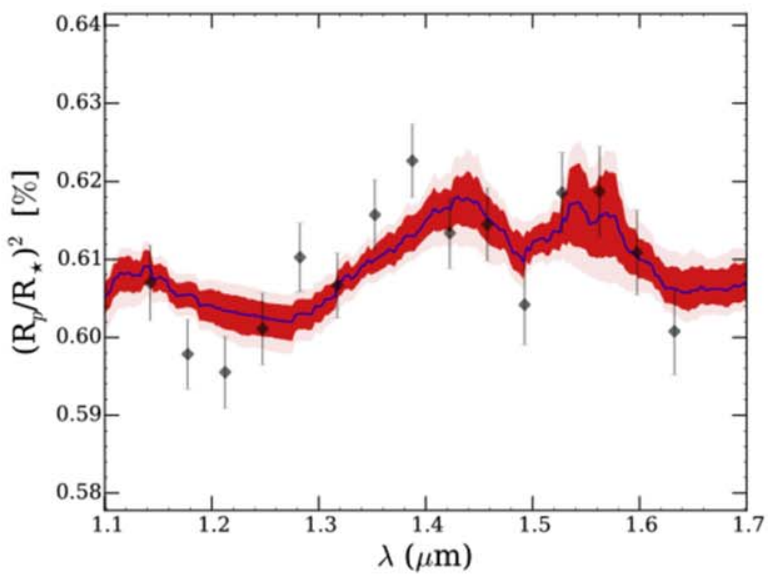

$\log \mathrm{HCN}=-3.35+1.75$

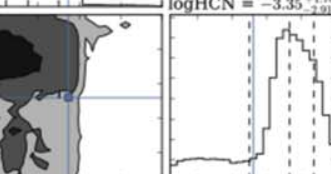

$\log \left(\mathrm{P}_{c}\right)=-2.02+20$

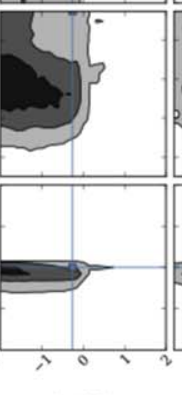

$\log (\mathrm{C} / \mathrm{O})$

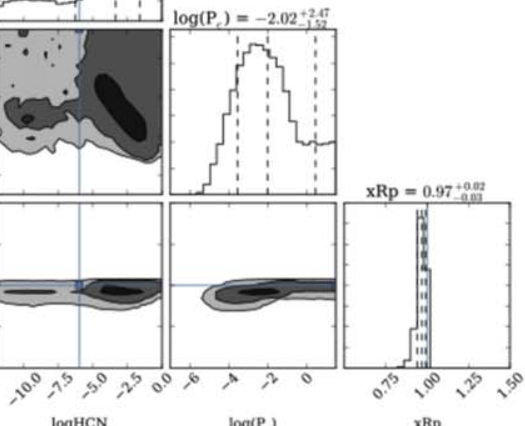

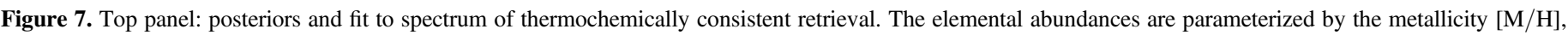

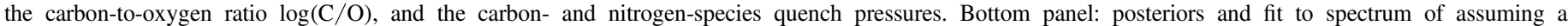
thermochemically consistent atmosphere with the addition of $\mathrm{HCN}$ as a free parameter. 


\section{MACDONALD \& MADHUSUDHAN}
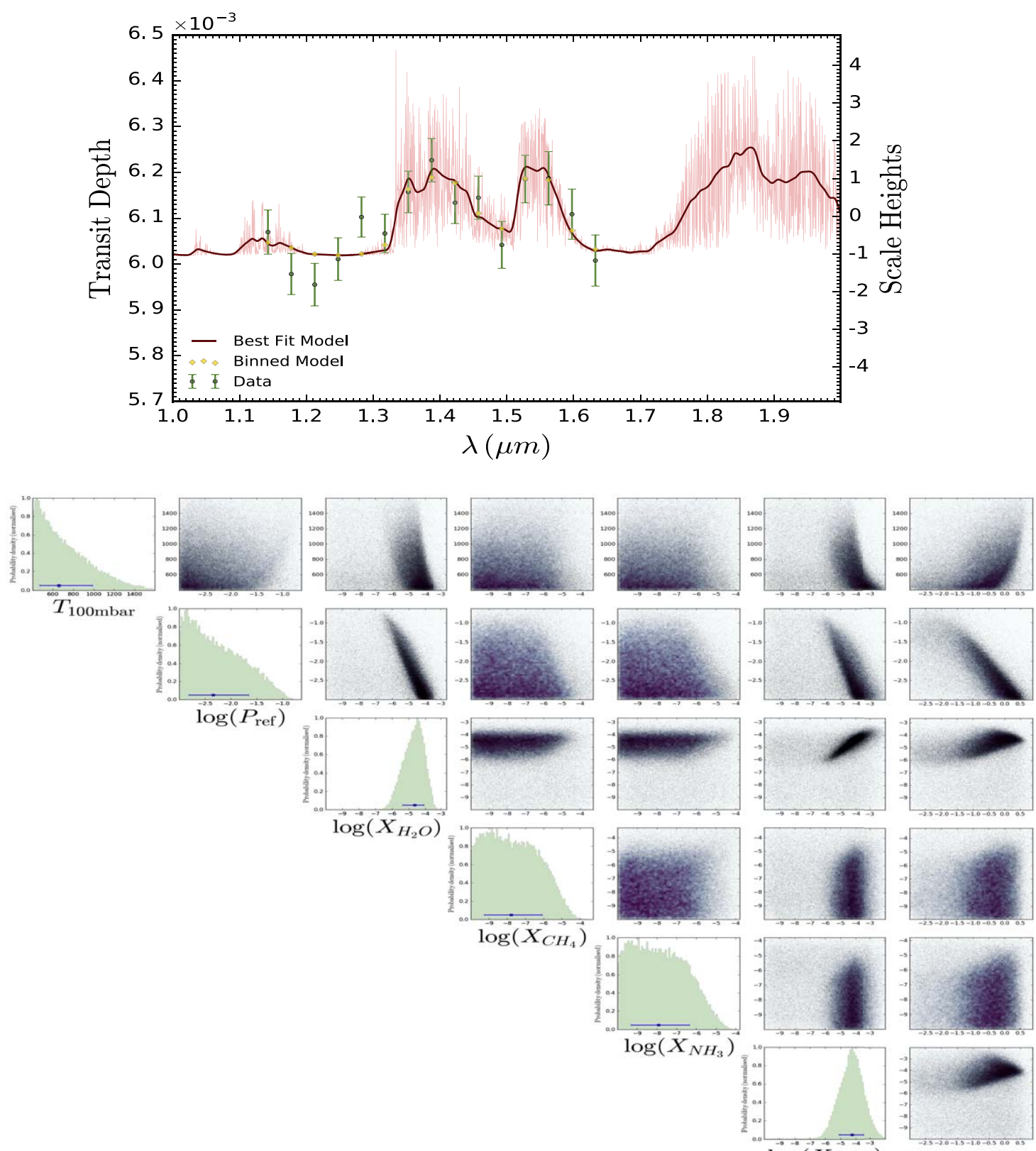

$\log \left(\vec{X}_{H C N}^{-\vec{H}}\right)$

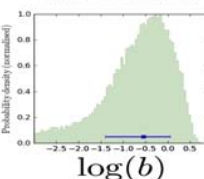

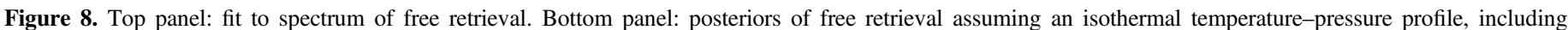
molecular opacities due to $\mathrm{H}_{2} \mathrm{O}, \mathrm{CH}_{4}, \mathrm{NH}_{3}$, and $\mathrm{HCN}$, and clouds as a uniform-in-altitude gray opacity. 


\section{WALDMANN}
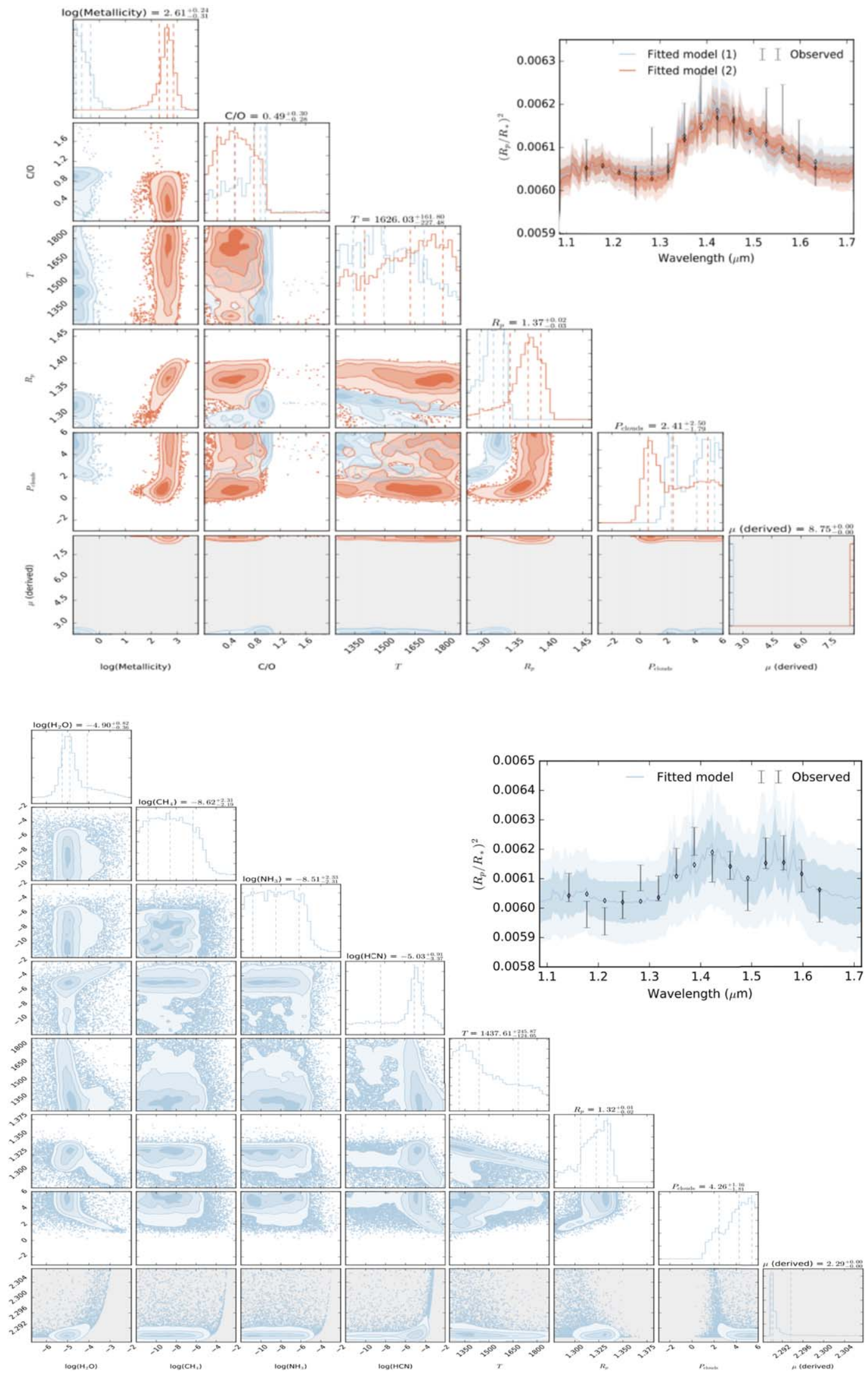

Figure 9. Top panel: posteriors and fit to spectrum of chemically consistent retrieval. The free parameters are the C/O ratio, atmospheric metallicity, planet radius, temperature, and cloud-top pressure. Bottom panel: posteriors and fit to spectrum of free retrieval with planet radius, temperature, cloud-top pressure, and abundances of $\mathrm{H}_{2} \mathrm{O}, \mathrm{CH}_{4}, \mathrm{CO}, \mathrm{CO}_{2}, \mathrm{NH}_{3}$, and $\mathrm{HCN}$ as free parameters. 


\section{ORCID iDs}

Brian M. Kilpatrick (iD https://orcid.org/0000-00034220-600X

Patricio E. Cubillos (10 https://orcid.org/0000-0002-1347-2600

Kevin B. Stevenson (iD https://orcid.org/0000-0002-7352-7941

Nikole K. Lewis (i) https://orcid.org/0000-0002-8507-1304

Hannah R. Wakeford (iD https://orcid.org/0000-00034328-3867

Ryan J. MacDonald (iD https://orcid.org/0000-0003-4816-3469

Nikku Madhusudhan (iD https://orcid.org/0000-0002-

4869-000X

Jasmina Blecic (iD https://orcid.org/0000-0002-0769-9614

Adam Burrows (iD https://orcid.org/0000-0002-3099-5024

Kevin Heng (1D https://orcid.org/0000-0003-1907-5910

Michael R. Line (i) https://orcid.org/0000-0002-2338-476X

Caroline V. Morley (10) https://orcid.org/0000-0002-4404-0456

Vivien Parmentier (iD https://orcid.org/0000-0001-9521-6258

Gregory S. Tucker (iD https://orcid.org/0000-0002-6954-6947

Ingo P. Waldmann (1D https://orcid.org/0000-0002-4205-5267

Jacob L. Bean (10) https://orcid.org/0000-0003-4733-6532

Joshua D. Lothringer (1D https://orcid.org/0000-0003-

3667-8633

Avi M. Mandell (i) https://orcid.org/0000-0002-8119-3355

\section{References}

Abel, M., Frommhold, L., Li, X., \& Hunt, K. L. C. 2012, JChPh, 136, 044319 Ackerman, A. S., \& Marley, M. S. 2001, ApJ, 556, 872

Agúndez, M., Venot, O., Iro, N., et al. 2012, A\&A, 548, A73

Barber, R. J., Strange, J. K., Hill, C., et al. 2014, MNRAS, 437, 1828

Benneke, B. 2015, arXiv:1504.07655

Berta, Z. K., Charbonneau, D., Désert, J.-M., et al. 2012, ApJ, 747, 35

Blecic, J. 2016, arXiv:1604.02692

Blecic, J., Harrington, J., \& Bowman, M. O. 2016, ApJS, 225, 4

Borysow, A. 2002, A\&A, 390, 779

Borysow, A., \& Frommhold, L. 1989, ApJ, 341, 549

Borysow, A., Frommhold, L., \& Moraldi, M. 1989, ApJ, 336, 495

Borysow, A., Jorgensen, U. G., \& Fu, Y. 2001, JQSRT, 68, 235

Borysow, J., Frommhold, L., \& Birnbaum, G. 1988, ApJ, 326, 509

Brown, T. M. 2001, ApJ, 553, 1006

Buchner, J., Georgakakis, A., Nandra, K., et al. 2014, A\&A, 564, A125

Burrows, A., Marley, M., Hubbard, W. B., et al. 1997, ApJ, 491, 856

Burrows, A., \& Sharp, C. M. 1999, ApJ, 512, 843

Cubillos, P., Harrington, J., Loredo, T. J., et al. 2017, AJ, 153, 3

Cubillos, P. E. 2016, arXiv:1604.01320

Cubillos, P. E. 2017, ApJ, 850, 32

de Wit, J., Wakeford, H. R., Gillon, M., et al. 2016, Natur, 537, 69

Deming, D., Wilkins, A., McCullough, P., et al. 2013, ApJ, 774, 95

Evans, T. M., Sing, D. K., Wakeford, H. R., et al. 2016, ApJL, 822, L4

Feroz, F., \& Hobson, M. P. 2008, MNRAS, 384, 449

Foreman-Mackey, D., Hogg, D. W., Lang, D., \& Goodman, J. 2013, PASP, 125,306

Fortney, J. J. 2005, MNRAS, 364, 649

Fortney, J. J., Marley, M. S., Saumon, D., \& Lodders, K. 2008, ApJ, 683, 1104 Fraine, J., Deming, D., Benneke, B., et al. 2014, Natur, 513, 526

Freedman, R. S., Lustig-Yaeger, J., Fortney, J. J., et al. 2014, ApJS, 214, 25

Freedman, R. S., Marley, M. S., \& Lodders, K. 2008, ApJS, 174, 504

Gelman, A., \& Rubin, D. B. 1992, StaSc, 7, 457

Greene, T. P., Line, M. R., Montero, C., et al. 2016, ApJ, 817, 17

Grimm, S. L., \& Heng, K. 2015, ApJ, 808, 182

Guillot, T. 2010, A\&A, 520, A27
Hedges, C., \& Madhusudhan, N. 2016, MNRAS, 458, 1427

Hellier, C., Anderson, D. R., Collier Cameron, A., et al. 2012, MNRAS, 426, 739

Heng, K., \& Kitzmann, D. 2017, arXiv:1702.02051

Hollis, M. D. J., Tessenyi, M., \& Tinetti, G. 2013, CoPhC, 184, 2351

Howe, A. R., \& Burrows, A. S. 2012, ApJ, 756, 176

Knutson, H. A., Benneke, B., Deming, D., \& Homeier, D. 2014, Natur, 505, 66

Kreidberg, L. 2015, batman: BAsic Transit Model cAlculatioN in Python, Astrophysics Source Code Library, ascl:1510.002

Kreidberg, L., Bean, J. L., Désert, J.-M., et al. 2014a, ApJL, 793, L27

Kreidberg, L., Bean, J. L., Désert, J.-M., et al. 2014b, Natur, 505, 69

Kreidberg, L., Line, M. R., Bean, J. L., et al. 2015, ApJ, 814, 66

Kuntschner, H., Bushouse, H., Kümmel, M., \& Walsh, J. R. 2009, WFC3 SMOV proposal 11552: Calibration of the G141 grism, Tech. Rep., 17

Lecavelier Des Etangs, A., Pont, F., Vidal-Madjar, A., \& Sing, D. 2008, A\&A, 481, L83

Lee, J.-M., Heng, K., \& Irwin, P. G. J. 2013, ApJ, 778, 97

Liddle, A. R. 2007, MNRAS, 377, L74

Line, M. R., Knutson, H., Deming, D., Wilkins, A., \& Desert, J.-M. 2013a, ApJ, 778, 183

Line, M. R., \& Parmentier, V. 2016, ApJ, 820, 78

Line, M. R., Stevenson, K. B., Bean, J., et al. 2016, AJ, 152, 203

Line, M. R., Wolf, A. S., Zhang, X., et al. 2013b, ApJ, 775, 137

Lodders, K. 2009, Exoplanet Chemistry (New York: Wiley-VCH)

MacDonald, R. J., \& Madhusudhan, N. 2017, arXiv:1711.00467

Mandel, K., \& Agol, E. 2002, ApJL, 580, L171

Marley, M. S., Gelino, C., Stephens, D., Lunine, J. I., \& Freedman, R. 1999, ApJ, 513, 879

Marley, M. S., Saumon, D., Guillot, T., et al. 1996, Sci, 272, 1919

Marley, M. S., Seager, S., Saumon, D., et al. 2002, ApJ, 568, 335

McCullough, P., \& MacKenty, J. 2012, Considerations for using Spatial Scans with WFC3, Tech. Rep., 8

McKay, C. P., Pollack, J. B., \& Courtin, R. 1989, Icar, 80, 23

Mordasini, C., van Boekel, R., Mollière, P., Henning, T., \& Benneke, B. 2016, ApJ, 832, 41

Morley, C. V., Fortney, J. J., Marley, M. S., et al. 2015, ApJ, 815, 110

Morley, C. V., Knutson, H., Line, M., et al. 2017, AJ, 153, 86

Moses, J. I., Madhusudhan, N., Visscher, C., \& Freedman, R. S. 2013, ApJ, 763,25

Moses, J. I., Visscher, C., Fortney, J. J., et al. 2011, ApJ, 737, 15

Parmentier, V., Fortney, J. J., Showman, A. P., Morley, C., \& Marley, M. S. 2016, ApJ, 828, 22

Richard, C., Gordon, I. E., Rothman, L. S., et al. 2012, JQSRT, 113, 1276

Rothman, L. S., Gordon, I. E., Babikov, Y., et al. 2013, JQSRT, 130, 4

Rothman, L. S., Gordon, I. E., Barbe, A., et al. 2009, JQSRT, 110, 533

Rothman, L. S., Gordon, I. E., Barber, R. J., et al. 2010, JQSRT, 111, 2139

, S., \& Madhusudhan, N. 2017, arXiv

Saumon, D., \& Marley, M. S. 2008, ApJ, 689, 1327

Sharp, C. M., \& Burrows, A. 2007, ApJS, 168, 140

Showman, A. P., Fortney, J. J., Lian, Y., et al. 2009, ApJ, 699, 564

Sing, D. K., Fortney, J. J., Nikolov, N., et al. 2016, Natur, 529, 59

Stevenson, K. B. 2016, ApJL, 817, L16

Stevenson, K. B., Désert, J.-M., Line, M. R., et al. 2014, Sci, 346, 838

Stevenson, K. B., Lewis, N. K., Bean, J. L., et al. 2016, PASP, 128, 094401

Swain, M. R., Line, M. R., \& Deroo, P. 2014, ApJ, 784, 133

Tennyson, J., \& Yurchenko, S. N. 2012, MNRAS, 425, 21

Tennyson, J., Yurchenko, S. N., Al-Refaie, A. F., et al. 2016, JMoSp, 327, 73

Tinetti, G., Tennyson, J., Griffith, C. A., \& Waldmann, I. 2012, RSPTA, 370, 2749

Toon, O. B., \& Ackerman, T. P. 1981, ApOpt, 20, 3657

Venot, O., Hébrard, E., Agúndez, M., et al. 2012, A\&A, 546, A43

Wakeford, H. R., Sing, D. K., Evans, T., Deming, D., \& Mandell, A. 2016, ApJ, 819, 10

Wakeford, H. R., Stevenson, K. B., Lewis, N. K., et al. 2017, ApJL, 835, L12

Waldmann, I. P. 2016, ApJ, 820, 107

Waldmann, I. P., Rocchetto, M., Tinetti, G., et al. 2015a, ApJ, 813, 13

Waldmann, I. P., Tinetti, G., Rocchetto, M., et al. 2015b, ApJ, 802, 107 